\title{
Edetic Acid
}

National Cancer Institute

\section{Source}

National Cancer Institute. Edetic Acid. NCI Thesaurus. Code C61742.

The acid form of edetate, a chelating agent with anti-hypercalcemic and anticoagulant properties. Edetic acid binds calcium and heavy metal ions, forming soluble stable complexes which are readily excreted by the kidneys. This results in a decrease in serum calcium levels. This agent is also used as an anticoagulant for blood specimens and is applied as a treatment of lead poisoning. 